\title{
Empirical Viscosity-Concentration Relationship for Polymer Solutions
}

\author{
CHEN SHOU-ZHONG \\ GuangZhou Institute of Rubber Industry, \\ GuangZhou, China.
}

(Received February 22, 1982)

\begin{abstract}
KEY WORDS Polymer Solution Viscosity / Concentration Dependence / Intrinsic Viscosity / Huggins Coefficient / Concentrated Solutions /
\end{abstract}

Viscometry is a very popular method for obtaining information on the size and structure of polymer molecules in solution. In order to obtain polymer parameters from viscosity measurement of solutions, it is necessary to establish a viscosityconcentration relationship.

The viscosity of a dilute solution of a neutral polymer may be expressed as a power series of concentration:

$$
\begin{gathered}
\eta_{\mathrm{r}} \equiv \eta / \eta_{0}=1+[\eta] c+k_{1}[\eta]^{2} c^{2} \\
+k_{2}[\eta]^{3} c^{3}+\cdots
\end{gathered}
$$

where $\eta_{0}$ and $\eta$ are, respectively, the solvent viscosity and the solution viscosity, $\eta_{\mathrm{r}}$ is the relative viscosity, $c$ is the concentration expressed in grams of polymer per unit volume of solution, and $[\eta], k_{1}$, and $k_{2}$ are constants independent of $c$. The linearterm coefficient $[\eta]$ is called the intrinsic viscosity which is one of the most important sources of information on the size and shape of polymer molecules. Thus, the method for evaluating $[\eta]$ has been intensively studied by many investigators since the beginning of polymer science.

The higher order terms in eq 1 naturally become insignificant at low concentrations. Thus, we may approximate eq 1 by either of the following forms:

$$
\begin{gathered}
\eta_{\mathrm{sp}} / c \equiv\left(\eta_{\mathrm{r}}-1\right) / c=[\eta]+k_{1}[\eta]^{2} c \\
\eta_{\mathrm{sp}} / c=[\eta]+k_{1}[\eta] \eta_{\mathrm{sp}}
\end{gathered}
$$

where $\eta_{\mathrm{sp}}$ is the specific viscosity. Equations 2 and 3 are, respectively, the Huggins and the Schulz-
Blaschke equations, ${ }^{1,2}$ and $k_{1}$ is usually referred to as the Huggins constant. These equations suggest that if a series of accurate data is available at sufficiently low concentrations, the plots of $\eta_{\mathrm{sp}} / c$ against $c$ and $\eta_{\mathrm{sp}}$ both give straight lines and [ $\left.\eta\right]$ is readily obtained from their intersections with the ordinate, $c=0$ or $\eta_{\mathrm{sp}}=0$. These procedures are, however, not generally practical, for the experimental uncertainty in the $\eta_{\mathrm{sp}} / c$ ratio becomes impractically large at "sufficiently" low concentrations. Thus, neither eq 2 nor 3 is strictly valid at most practical concentrations, and a nonlinear extrapolation of the plots of $\eta_{\mathrm{sp}} / c$ against $c$ and $\eta_{\mathrm{sp}}$ is needed for evaluating $[\eta]$.

It is widely recognized that an exponent-type equation is often useful for describing a physical quantity when the power series equation becomes impractical. In the present case of polymer solution viscosity, the old Baker equation ${ }^{3}$ is an example:

$$
\eta_{\mathrm{r}}=(1+a c)^{k}
$$

where $a$ and $k$ are constants. Sakurada ${ }^{4}$ has shown that this equation is valid at relatively high concentrations with $k=3 \sim 4$ for polystyrene, poly(methyl methacrylate) and polyisobutyrene and that $[\eta]$ is obtainable according to $[\eta]=a k$. At sufficiently high concentrations where $a c \gg 1$, eq 4 yields

$$
\eta_{\mathrm{r}}^{1 / k}=a c
$$

We note that this equation represents a concentration scaling for the viscosity. ${ }^{5}$ 


\section{S. Z. CHEN}

Table I. Viscosity data used for testing the validity of eq 6

1. Polystyrene $(M=600,000)$ in toluene at $30^{\circ} \mathrm{C}$. Data of Weissberg, Simha, and Rothman. ${ }^{6}$

\begin{tabular}{lrrrr}
\hline \multicolumn{1}{c}{$c^{\mathrm{a}}$} & \multicolumn{1}{c}{$\eta_{\mathrm{r}}$} & \multicolumn{1}{c}{$\eta_{\mathrm{sp}}$} & $\eta_{\mathrm{sp}} / c^{\mathrm{a}}$ & $\eta_{\mathrm{sp}} / \eta_{\mathrm{r}}^{0.28}$ \\
\hline 0.091 & 1.199 & 0.199 & 2.1868 & 0.1891 \\
0.3384 & 1.872 & 0.872 & 2.5768 & 0.7316 \\
0.5602 & 2.616 & 1.616 & 2.8847 & 1.2345 \\
0.6367 & 2.922 & 1.922 & 3.0187 & 1.4235 \\
0.7236 & 3.336 & 2.336 & 3.2283 & 1.6671 \\
0.8469 & 3.872 & 2.872 & 3.3912 & 1.9659 \\
0.9196 & 4.205 & 3.205 & 3.4852 & 2.1437 \\
1.1824 & 5.653 & 4.653 & 3.9352 & 2.8648 \\
1.2671 & 6.143 & 5.143 & 4.0589 & 3.0936 \\
1.3795 & 6.954 & 5.954 & 4.3161 & 3.4593 \\
1.6554 & 9.065 & 8.065 & 4.8719 & 4.3505 \\
1.8512 & 10.747 & 9.747 & 5.2652 & 5.0131 \\
2.0692 & 13.033 & 12.033 & 5.8153 & 5.8636 \\
2.2626 & 15.206 & 14.206 & 6.2786 & 6.6299 \\
2.5454 & 19.008 & 18.008 & 7.0747 & 7.8952 \\
2.909 & 24.748 & 23.748 & 8.1636 & 9.6702 \\
3.3939 & 39.799 & 33.799 & 9.9587 & 12.5102 \\
\hline
\end{tabular}

2. Poly(4-vinylbiphenyl $(M=706,500)$ in benzene at $30^{\circ} \mathrm{C}$. Data of Utracki, Simha, and Eliezer ${ }^{8}$

\begin{tabular}{ccclc}
\hline \multicolumn{1}{c}{$c^{\mathrm{a}}$} & \multicolumn{1}{c}{$\eta_{\mathrm{r}}$} & \multicolumn{1}{c}{$\eta_{\mathrm{sp}}$} & \multicolumn{1}{c}{$\eta_{\mathrm{sp}} / c^{\mathrm{a}}$} & $\eta_{\mathrm{sp}} / \eta_{\mathrm{r}}^{0.28}$ \\
\hline 0.2333 & 1.234 & 0.234 & 1.003 & 0.2206 \\
0.2979 & 1.304 & 0.304 & 1.0205 & 0.2822 \\
0.4179 & 1.441 & 0.441 & 1.0553 & 0.3981 \\
0.5268 & 1.577 & 0.577 & 1.0953 & 0.5079 \\
1.4943 & 3.067 & 2.067 & 1.3833 & 1.5103 \\
1.756 & 3.604 & 2.604 & 1.4829 & 1.8186 \\
2.317 & 4.84 & 3.84 & 1.6573 & 2.4693 \\
2.9324 & 6.449 & 5.449 & 1.8582 & 3.2334 \\
3.7595 & 9.147 & 8.147 & 2.167 & 4.3837 \\
4.6617 & 12.817 & 11.817 & 2.5349 & 5.7853 \\
4.8344 & 13.747 & 12.747 & 2.6367 & 6.1194 \\
6.1664 & 21.54 & 20.54 & 3.331 & 8.6954 \\
8.1869 & 37.712 & 36.712 & 4.4842 & 13.286 \\
11.123 & 84.271 & 83.271 & 7.4864 & 24.06 \\
\hline
\end{tabular}

a $c$ is expressed in gram per $100 \mathrm{~cm}^{3}$ of solution.

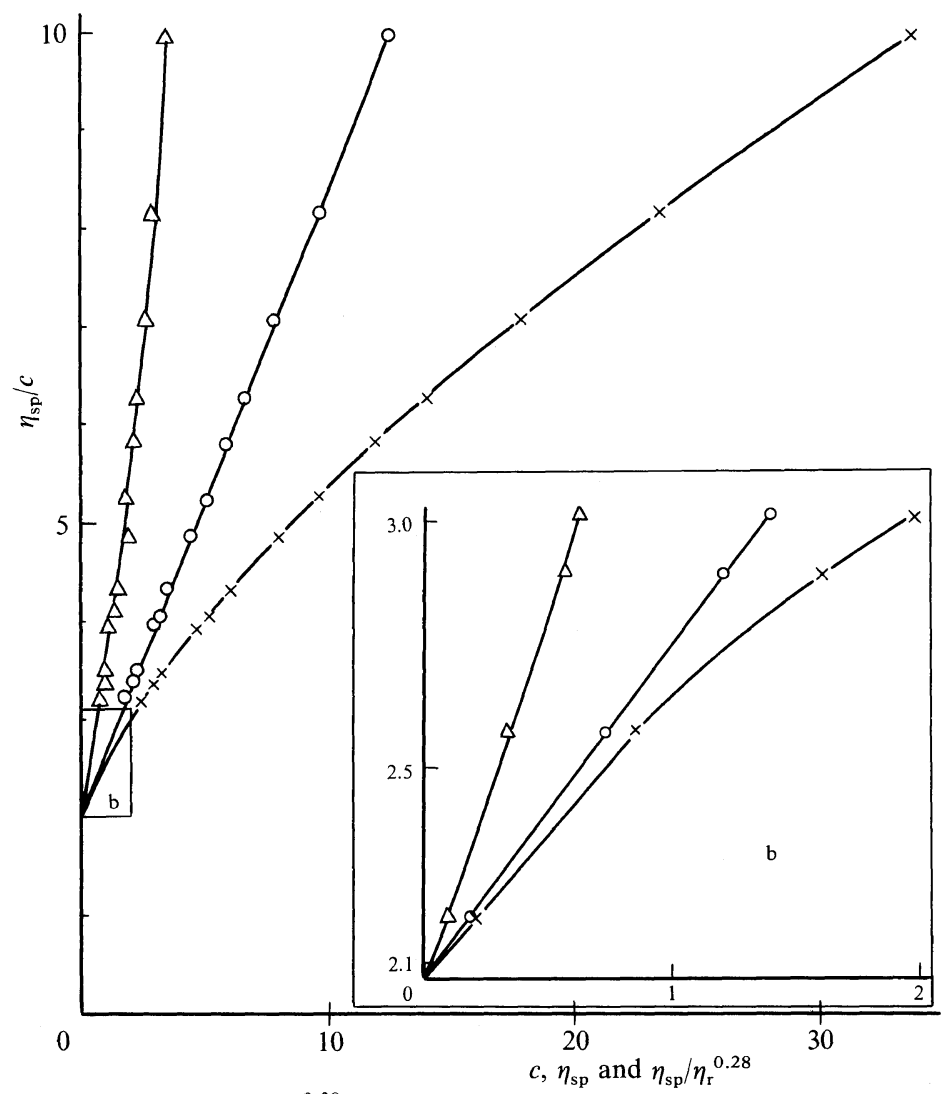

Figure 1. $\eta_{\mathrm{sp}} / c$ vs. $c, \eta_{\mathrm{sp}}$, and $\eta_{\mathrm{sp}} / \eta_{\mathrm{r}}{ }^{0.28}$ plots for polystyrene $(M=600,000)$ in toluene at $30^{\circ} \mathrm{C}: \triangle, \eta_{\mathrm{sp}} / c v s$. $c ; \bigcirc, \eta_{\mathrm{sp}} / c v s . \eta_{\mathrm{sp}} / \eta_{\mathrm{r}}{ }^{0.28} ; \times, \eta_{\mathrm{sp}} / c v s . \eta_{\mathrm{sp}}$. Data of Weissberg, Simha, and Rothman. ${ }^{6}$ 


\section{NEW EMPIRICAL EQUATION}

As mentioned above, eq 3 is valid at sufficiently low concentrations, while eq 5 is valid at sufficiently high concentrations. Thus, we may expect that a hybrid form of these two equations is applicable over a very wide range of concentration:

$$
\eta_{\mathrm{sp}} / c=[\eta]+\left(k_{1}[\eta] \eta_{\mathrm{sp}} / \eta_{\mathrm{r}}{ }^{\gamma}\right)
$$

This equation reduces to eq 2 or 3 when $c$ approximate to zero or $\eta_{\mathrm{r}}$ to unity, and it reduce to following equation

$$
\eta_{\mathrm{r}}{ }^{\gamma}=k_{1}[\eta] c
$$

when the first term $[\eta]$ is negligible in comparison with the second term.

Tests of eq 6 were made using the two series of data shown in Table I, which were obtained by Weissberg, Simha, and Rothman ${ }^{6,7}$ for solutions of polystyrene in toluene and by Utracki, Simha, and Eliezer ${ }^{8,7}$ for solutions of poly(4-vinylbiphenyl) in benzene. These data are plotted in Figures 1 and 2 against $c, \eta_{\mathrm{sp}}$, and $\eta_{\mathrm{sp}} / \eta_{\mathrm{r}}{ }^{0.28}$, each corresponding to eq 2,3 , and 6 . The $\gamma$ value of 0.28 was chosen so as to make the plot as straight as possible. As can be readily seen from these figures, only the present equation 6 yields straight lines throughout the entire range from sufficiently low concentration extend to $c=10 \mathrm{~g} 100 \mathrm{~cm}^{-3}$, and $\eta_{\mathrm{r}}=100$.

Also, similar results were obtained, though not reproduced here, for solutions of polystyrene in methyl ethyl ketone, poly(1-vinylnaphthalene) in benzene, and poly(2-vinylnaphthalene) in benzene. The best fit value of $\gamma$ was again 0.28 for each system. Thus, the present analysis seems to suggest that $\eta_{\mathrm{r}}$ of concentrated solutions is generally proportional to $([\eta] c))^{3,5}$

The two constants $[\eta]$ and $k_{1}$ in eq 6 are essentially the parameters characteristic of dilute solutions and are obtainable from the viscosity data at low concentrations. Hence, if $\gamma$ is a universal constant, we may with the aid of eq 6 , predict the viscosity $\eta_{\mathrm{r}}$ for concentrated solutions from the data for dilute solutions.

Acknowledgement. The author is very grateful to Professor Michio Kurata of Kyoto University for kindly preparing the sample used in this experiment

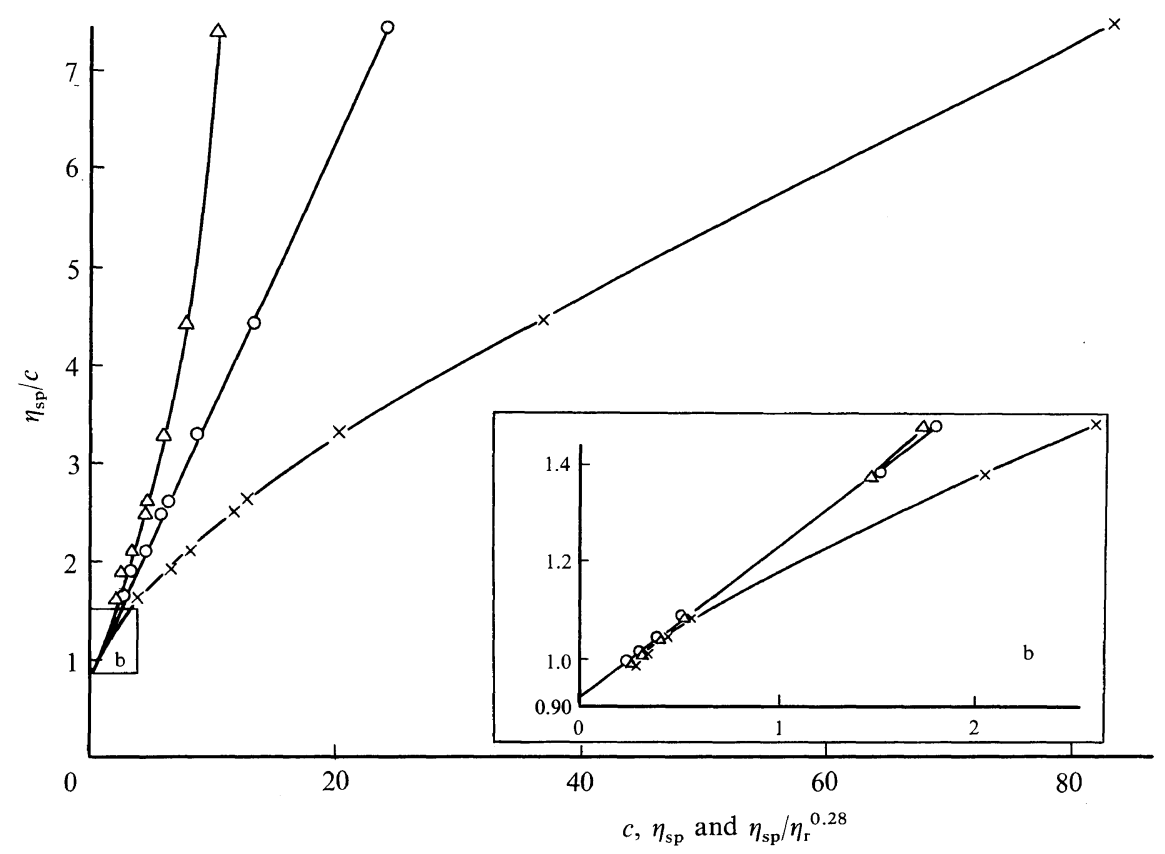

Figure 2. $\eta_{\mathrm{sp}} / c v s . c, \eta_{\mathrm{sp}}$, and $\eta_{\mathrm{sp}} / \eta_{\mathrm{r}}^{0.28}$ plots for poly(4-vinyl biphenyl) $(M=706,500)$ in benzene at $30^{\circ} \mathrm{C}$. $\triangle, \eta_{\mathrm{sp}} / c$ vs. $c ; \mathrm{O}, \eta_{\mathrm{sp}} / c v s . \eta_{\mathrm{sp}} / \eta_{\mathrm{r}}{ }^{0.28} ; \times, \eta_{\mathrm{sp}} / c v s, \eta_{\mathrm{sp}}$. Data of Utracki, Simha, and Eliezer. ${ }^{8}$ 


\section{S. Z. CHEN}

and for his valuable comments.

\section{REFERENCES}

1. M. L. Huggins, J. Am. Chem. Soc., 64, 2716 (1942).

2. G. V. Schulz and F. Blaschke, J. Prakt. Chem. [2], 158, 136 (1941).

3. F. Baker, J. Chem. Soc., 103, 1653 (1913).

4. I. Sakurada, Kobunshi Kagaku, 2, 253, 260, 268
(1945).

5. P.-G. de Gennes, "Scaling Concepts in Polymer Physics," Cornell Univ. Press, Ithaca and London, 1979.

6. S. G. Weissberg, R. Simha, and S. Rothman, J. Res. Natl. Bur. Stand., 47, 289 (1951).

7. See also, A. Rudin and G. B. Strathdee, J. Paint Technol., 46, 33 (1974).

8. L. Utracki, R. Simha, and N. Eliezer, Polymer (London), 10, 43 (1969). 